\title{
Einstein, interaktiv und zum Anfassen
}

\section{Oder: die drohende Auflösung des Museums?}

\author{
Christian Sichau
}

Anke te Heesen hat in ihrem Beitrag zum Forum im Heft 4/2008 dieser Zeitschrift einige ermutigende Erfolge von Ausstellungen auf dem Gebiet der Wissenschafts- und Technikgeschichte aus den letzten Jahren angeführt. Gleichzeitig betont sie den prekären Status vieler Universitätssammlungen und -museen. Sie verweist darauf, dass uns die materielle und vielfach nur noch elektronische Hinterlassenschaft der zeitgenössischen Wissenschaft und Technik vor enorme Aufgaben stellt. Was können wir davon wirklich noch langfristig vor dem Verfall bewahren? Die Probleme, die sie anführt, sind bereits enorm und erfordern große Anstrengungen. Ich kann als Kurator eines großen deutschen Wissenschafts- und Technikmuseums diesen Ausführungen nur zustimmen, sie bedürfen sogar noch einer pessimistischen Zuspitzung. Im Mittelpunkt steht dabei eine zu konstatierende grundsätzliche Entwicklung in der Museumswelt, die in ihrer Tragweite noch nicht ausreichend zur Kenntnis genommen wurde. In pointierter Form hat bereits der Oxforder Instrumentenhistoriker und Museumsleiter Jim Bennett auf diese Situation hingewiesen: „History of science has no divine right to rule just because the objects in the museum are old." Sowohl hinsichtlich eines inzwischen viel breiter gewordenen Aufgabenspektrums als auch mit Blick auf die gestaltenden und Einfluss nehmenden Akteure stellen Wissenschafts- und Technikgeschichte für Museen heute bestenfalls nur einen unter vielen Bezugspunkten dar. Die Konsequenzen dieser Entwicklung sind sorgfältig zu analysieren. Dass eine solche Analyse dringend erforderlich ist, zeigt auch ein im Physik-Journal im März 2008 veröffentlichter Appell des Wissenschaftshistorikers Klaus Hentschel unter der Überschrift: „Bitte nicht wegwerfen!" Sein an die Physikergemeinde gerichteter Aufruf, Quellen der Wissenschaftsgeschichte an Archive und Museen weiterzugeben, greift in der gegenwärtigen Situation der Museen jedenfalls deutlich zu kurz. Denn der Sammel- und Bewahrungsauftrag muss im veränderten Aufgabenspek- 
trum der Museen heute als gefährdet aufgefasst werden. Aus meiner Sicht ist auf zwei bemerkenswerte Entwicklungen hinzuweisen: Zum einen auf eine in Konkurrenz zu den klassischen Wissenschafts- und Technikmuseen stehende Ausstellungs- und Vermittlungsdynamik, die in ihren Auswirkungen noch zu wenig beachtet wurde; zum anderen, daran anknüpfend, eine zugespitzte Diskussion der Sammlungsproblematik.

Das Einsteinjahr 2005 hat uns anschaulich vorgeführt: Interaktion und Medialität, Erlebnis und Spiel stehen im Vordergrund; einleuchtende Demonstrationen und einfach, das heißt verständlich aufgebaute physikalische Experimente sind gegenüber einer gesamten Ausbreitung aller bis heute erforschten Fakten über Einsteins Leben zu bevorzugen; zu viele Dokumente und Fotos, noch unterstützt durch lange wissenschaftliche Texte, können kaum noch mit dem Interesse eines großen Publikums rechnen. In solchen Selbstdarstellungen zahlreicher Aktivitäten, die im Einsteinjahr die Masse erreichen sollten, wurde bewusst ein Kontrastbild zu vermeintlich traditionellen Museumsausstellungen konstruiert, die abschätzig zu einer „konventionellen Schau aus statischen Exponaten mit erklärenden Texten" deklariert wurden. Bemerkenswerterweise waren solche Äußerungen und Zerrbilder zum Teil auch in den Museen selbst zu hören. Diese öffentlichkeitswirksamen Maßnahmen mögen weniger im Blickfeld der wissenschafts- und technikhistorischen Zunft gewesen sein als etwa die große Berliner Einstein-Ausstellung im Kronprinzenpalais. In Berlin ebenso wie in der im Deutschen Museum gezeigten kleinen Einstein-Schau war der Versuch unternommen worden, in der Konzeption die Vermittlung neuester wissenschaftshistorischer Forschungsergebnisse tragend zur Geltung zu bringen, während andernorts durchaus auch das Programmieren mit Lego-Robotern als Einstein-Aktivität im wahrsten Sinne des Wortes verkauft wurde, mit der Begründung: „Diese Einführung in die Zukunftstechnologie des Konstruierens und Programmierens von Robotern hätte sicher auch Einstein begeistert."

Manche der in Berlin und München zu Recht kritisierten fachlichen Mängel sind darauf zurückzuführen, dass auch diese Ausstellungen sich eben mit jenen anderen Einstein-Aktivitäten messen lassen mussten, die sich so vollkommen entgegengesetzt positionierten. Diese andere Ausstellungs- und Vermittlungswelt ist mächtig, vor allen Dingen in den Medien und sie finanziert sich aus denselben öffentlichen und privaten Töpfen, die auch für wissenschaftshistorisch fundierte Aktivitäten in Anspruch genommen werden. Was in dieser Konkurrenz zählt, ist in diesem Fall etwa eine offiziell vom Bundesministerium für Bildung und Forschung in Auftrag gegebene Evaluierung, in der die Besucherinnen und Besucher etwa danach befragt werden, ob ihnen die Ausstellung wissenschaftliche Theorien oder Erkenntnisse anschaulich vermitteln konnte. Hier geht es um Physik anstelle von Physikgeschichte, und die Intention wird offen zum Ausdruck gebracht: 
„Hat Sie die Veranstaltung darin bestätigt oder bestärkt, eine Ausbildung/ Studium im naturwissenschaftlich-technischen Bereich zu beginnen?" Die Frage verweist auf ein prominentes Argument, welches Ausstellungen, Museen und Vermittlungsaktivitäten im Bereich von Wissenschaft und Technik heute aufgreifen müssen, um sich zu legitimieren und Fördermittel zu erhalten. Denn: Geld ist nicht alles, aber ohne Geld geht auch im Museum und in der Ausstellungswelt nichts. Mit normalen Haushaltsmitteln eines Museums lassen sich weder Sonderausstellungen noch größere Sammelaktivitäten finanzieren. Mit solchen Evaluationskriterien und den bereits erwähnten Werbebotschaften der verschiedensten Institutionen und Einrichtungen zum Einsteinjahr wurden Positionierungen vorgenommen und Erwartungshaltungen beziehungsweise Meinungen in der Öffentlichkeit erzeugt, die die Museen unter Druck setzen. Der Auftrag, vor allem zu einer Erhöhung der Studierendenzahlen beizutragen, wird dabei unmittelbar an eine spezielle Form gekoppelt, in welcher Weise dies zu geschehen hat. Dies geschieht ohne Diskussion, ob ein solcher Auftrag an die Museen nicht gar $\mathrm{zu}$ einseitig formuliert sei oder ob die Fokussierung auf Spaß und Unterhaltung tatsächlich das gewünschte Ergebnis bringe. Und es geschieht auch ungeachtet aller empirischen Untersuchungen über die Herausbildung von Studienneigungen, die Akzeptanz von Wissenschaft und Technik oder die Wirkungen von Ausstellungen.

Das Einsteinjahr kann hier nur als ein herausragendes, weil großes Aufsehen erregendes und Beachtung findendes Beispiel dienen. Es war letztlich nur ein weiteres jener Wissenschaftsjahre, die das Bundesministerium für Bildung und Forschung (BMBF) seit dem Jahr 2000 zusammen mit der Initiative Wissenschaft im Dialog (WiD) veranstaltet. Mit dem plakativen, in Zeitungsanzeigen großformatig abgedruckten Slogan „ $\mathrm{D}=\mathrm{mc}^{2 “}$, in dem man tatsächlich grafisch das Autokennzeichen für Deutschland („D“) verwendete, wurde im Einsteinjahr der unmittelbare politische wie ökonomisch begründete Kontext sichtbar, der diese Wissenschaftsjahre begleitet. Während die wissenschafts- und technikhistorische Forschung, da ist Anke te Heesen zuzustimmen, gerade durch ihre Einbindung in spektakuläre kulturhistorische Ausstellungen durchaus an öffentlicher Wahrnehmung gewonnen hat und auf diese Weise den akademischen Elfenbeinturm verlassen konnte, bleibt zu fragen, ob sich das Fach damit zufrieden geben sollte, nun im hübsch gestalteten, kleinen Vorgarten des Turms angekommen zu sein, sozusagen im feuilletonistischen Biotop der überregionalen Presse, aber anderen den Marktplatz zu überlassen. Denn was dort derzeit geschieht, hat gravierende Konsequenzen für die Museen. Ähnlich wie bei den Wissenschaftsjahren versteht sich die enorm wachsende Anzahl regionaler und lokaler sogenannter „Public Outreach“-Aktivitäten, unter Einbindung aktiver Fachwissenschaftler der Universitäten und Forschungseinrichtungen, meist als gegenwartsbezogen, in der Verkaufsrhetorik und Werbesprache gar „zu- 
kunftsorientiert", wobei eine (bewusste) Abgrenzung von Museen und ihren historischen Sammlungen festzustellen ist. Diese Aktivitäten sind eingebunden in die Imagepflege der Universitäten in ihrem Konkurrenzkampf um Studierende. Geschichte und somit auch historische Artefakte geraten dabei häufig aus dem Blick oder verkommen zum folkloristischen Beiwerk. Diese Verdrängung reicht heute bis in die Museen selbst hinein, wenn von deren Ausstellungsräumen im wahrsten Sinne des Wortes Besitz ergriffen wird, um dort aktuelle Forschung zu präsentieren. Universitätssammlungen und Museen haben hier zukünftig eine große Verantwortung, um vor Ort für das historische Bewusstsein zu sorgen und diesem Verdrängungsprozess entgegenzutreten.

Allerdings werden Universitätssammlungen und -museen in der Regel selbst inneruniversitär als Teil des „Public Outreach“ verstanden. Sie sind nicht frei und durch höhere Vernunft eingerichtete Institutionen, die diesem Markt und seinen Gesetzmäßigkeiten entzogen sind. Kann man unabhängige Wissenschafts- oder Technikgeschichte in einem Universitätsmuseum machen, wenn man Teil dieses Marktes, Teil der Presse- und Öffentlichkeitsarbeitsstelle der Universität ist? Welche schleichenden Veränderungen hier zu beobachten sind, zeigt beispielhaft die kürzlich veranstaltete PlanckAusstellung im Deutschen Technikmuseum in Berlin. Praktisch ungefiltert und unbearbeitet, bis hin zu der durch die Corporate Identity vorgegebenen Farbgebung, präsentierten sich hier in einem Teil der Ausstellung einzelne Institute der Max-Planck-Gesellschaft (MPG). Eine Ausstellung, deren Anlass eigentlich der 150. Geburtstag von Max Planck war und die sich in einem öffentlichen Museum (nicht etwa im Foyer eines Instituts der MPG) seiner wissenschaftlichen Tätigkeit widmete, mutierte ganz ungeniert zur Eigenwerbung des Geldgebers. Vermutlich sollte man heute die mangelnde Unabhängigkeit angesichts omnipräsenter Werbe- und Marketingmaßnahmen kaum mehr anstößig finden. Sie ist aber ein allgemeines Charakteristikum des Marktes, in dem auch Ausstellungen mit wissenschafts- und technikhistorischem Anspruch agieren.

Das gilt nicht nur für die Universitätssammlungen und -museen, sondern auch für das Deutsche Museum. Hentschels Aufruf, alles zu sammeln und zu bewahren, muss ich daher zum gegenwärtigen Zeitpunkt negativ beantworten: Nein, wir im Deutschen Museum prüfen und beraten nicht gerne, welche Geräte und Instrumente wir aus einem Labor übernehmen wollen. Auch das Abholen ist nicht per se eingeschlossen. Denn wir sind ebenfalls nicht durch eine höhere Vernunft mit einer sicheren Existenz ausgestattet, frei und unabhängig in unseren Entscheidungen, was wir warum sammeln. Die Museumsdepots sind voll. Dies gilt fast überall, auch international. Der Alltag ist von der Ablehnung eines angebotenen Objektes bestimmt und nicht von seiner Aufnahme in den Sammlungsbestand. Meist mag es gute wissenschafts- oder technikhistorische Gründe geben, ein Objekt bewahren zu 
wollen. Trotzdem werde ich als Kurator „Nein“ sagen müssen. Diese negative Antwort trifft besonders jene Objekte der zweiten Hälfte des 20. Jahrhunderts hart, die heute noch in den Laboratorien und Abstellkammern stehen und die aus technik- und wissenschaftshistorischer Sicht als bewahrenswert gelten können. In der Konkurrenz um Ressourcen innerhalb der Museen gewinnt nur noch das Objekt, welches es möglichst rasch in eine Ausstellung schafft. Wir sammeln für Ausstellungen, nicht um des Bewahrens willen. Denn Ausstellungen sind sichtbar und somit geeignet, wiederum neue Ressourcen einzuwerben. Für die historischen Objekte und ihre drohende $\mathrm{Zu}$ kunft auf dem Schrottplatz ist es ein eklatanter Einschnitt, wenn Museen auf ihren Bildungsauftrag reduziert werden und ihre Aufgabe als Orte des Sammelns und Bewahrens eines wissenschaftlich-technischen Kulturerbes angesichts knapper werdender Ressourcen stärker an gegenwartsbezogene Ausstellungen koppeln müssen. Objekte werden so unter Umständen abgelehnt - und damit entsorgt -, weil sie sich unglücklicherweise gerade in diesem Moment in kein Ausstellungsprojekt einfügen. Auch werden sie unter Umständen abgelehnt, weil sie momentan für nicht ausstellungsgeeignet erachtet werden. Gerade Objekte der Zeitgeschichte sind bedroht, denn sie können weder für eine nostalgische Rückschau in eine ferne Vergangenheit dienen noch stehen sie für die heutige Forschung und Wissenschaft. Sie erscheinen auch weniger spektakulär als Artefakte der Kunst- oder Wunderkammern der Renaissance. Die Anzahl elektronischer Instrumente in grauen Kisten etwa, die sich gleichzeitig in einem Ausstellungsraum befinden können, ist doch ziemlich begrenzt. Hinzu kommt die wachsende multimediale und inszenatorische Gestaltung vieler Expositionen, die die Anzahl der präsentierten Objekte signifikant reduziert.

Historische Bedeutung und Relevanz eines Objekts werden demgegenüber als Kriterien des Sammelns deutlich abgewertet. Dass diese sich, wie die historische Forschung gezeigt hat, oft gar erst im Nachhinein bestimmen lassen, wird dabei überhaupt nicht zur Kenntnis genommen. In Museen werden derzeit verschiedene Strategien diskutiert, mit dieser Problematik unter den gegebenen Randbedingungen zu Recht zu kommen. Ein Ansatz lässt sich als Flucht ins Lokale charakterisieren: Die Objekte müssen ihre (historische) Bedeutung vor Ort haben, wo die Besucherinnen und Besucher herkommen; übergeordnete Kriterien verlieren dabei an Bedeutung. Für das einzelne Museum mag dies ein sinnvolles Auswahlkriterium des Sammelns sein, aber kann die Summe der lokal bewahrten Dinge am Ende ein Bild vom Ganzen geben? Ein zweiter, weit verbreiteter Ansatz ist das exemplarische Sammeln. Auch dies mag im Einzelfall eine geeignete Strategie sein, um die Fülle der Angebote zu bewältigen. Doch auch dabei droht vieles verloren zu gehen. „Ein weiteres Elektronenmikroskop? Nein, danke. Wir haben schon eines. Es stammt zwar aus einer anderen Zeit und von einem anderen Hersteller, es ist auch anders konstruiert - doch uns genügt ein Elektronenmikroskop. Mehr 
können wir sowieso nicht ausstellen“, bin ich als Kurator geneigt zu antworten. Es scheint aus dieser Logik nur zu konsequent, dass bereits viele Museen mit dem „Entsammeln“ begonnen haben, wie diese Praxis bezeichnet wird. Wenngleich bekanntermaßen zum Sammeln auch das Wegwerfen gehört, erscheint damit eine Entwicklung in Gang gesetzt worden zu sein, die noch völlig ungeahnte Folgen hat, nicht nur für die objekthistorische Forschung im engeren Sinne, sondern auch für das allgemeine Studium inzwischen vergangener Wissenschaftspraktiken.

Während also das Ausstellungswesen boomt, sind Kernaufgaben des Museums wie das Sammeln, Bewahren und Forschen gefährdet. Denn die Bewahrung von Objekten in den Depots ist unsichtbar und verschlingt Ressourcen. Dafür Mittel und Unterstützung zu erhalten ist ungleich schwieriger. Voraussetzung wäre, das Bewahren sichtbar zu machen, etwa indem die in den Depots der Museen lagernde materielle Kultur der Wissenschaften und Technik durch Forschung und Publikationen ans Tageslicht gebracht würde. Doch entgegen den Beteuerungen und Ankündigungen blieb der erhoffte Ansturm der wissenschafts- und technikhistorischen Fachleute auf die Museumssammlungen bisher aus. Weiterhin werden Studien zu Instrumenten oder Instrumentenmachern publiziert, ohne dass museale Sammlungen eingehend konsultiert worden wären. Wenn die Gesellschaft für Technikgeschichte auf ihrer Jahrestagung 2008 die Frage stellt: „Wo steht die Technikgeschichte?", dann vermisse ich etwa die Frage nach der Bedeutung und Rolle der Museen für und in der Technikgeschichte. Dabei steht gerade die Technikgeschichte vor enormen Herausforderungen, was das zukünftige Sammeln und Bewahren angeht. Die wissenschafts- und technikhistorische Zunft müsste sich hier insgesamt stärker einbringen, denn die Fragen, um die es geht, sind nicht einfach zu beantworten, und die Museen sind als Institutionen strukturell und personell kaum mehr in der Lage, die Probleme alleine zu bewältigen. Umfassend ist in der Museumsszene zu spüren, „wie schwer wir alle uns mit dem Sammeln tun“. Dies konstatierte Wolfgang Kos vom Museum Wien in einem Überblick zur Sammlungspolitik als drängender Aufgabe dieser Institutionen auf dem österreichischen Museumstag 2007. Zugleich gab er das Motto aus: „Weitersammeln. Und zwar möglichst intelligent, engagiert und überlegt." Es sollte offensichtlich sein, dass hier Diskussionsprozesse angesprochen sind, die auch die Beteiligung der Vertreterinnen und Vertreter der Wissenschafts- und Technikgeschichte erfordern, die außerhalb der Museen tätig sind. Dies gilt gerade dann, wenn man Kos folgt, der bereits 2002 ein „Beobachtungsloch, [...] eine gefährlich weit geöffnete Schere zwischen Depot und jüngster Vergangenheit" feststellte. Die wissenschafts- und technikhistorische Forschung müsste also nicht nur die bereits in den Museen gesammelten materiellen Quellen in den Blick nehmen, sondern sich darüber hinaus zunehmend mit der Zukunft des Sammelns auseinandersetzen. 
Schließlich würde das Bewahren auch dann sichtbar werden, wenn Unbekanntes hervorgeholt und in temporären Ausstellungen präsentiert werden kann. Doch allzu oft finden wir in Ausstellungen lediglich das, was bereits bekannt ist, neu arrangiert, neu in Szene gesetzt, neu komponiert. Selten handelt es sich wirklich um bislang noch nicht gezeigte historische Exponate. Im ewigen Ausstellungspaternoster fahren letztlich doch nur sehr wenige, mehr oder minder bekannte Objekte umher, werden kurzzeitig entnommen und später wieder eingefügt, während die große Mehrzahl in den Sammlungen kaum zur Kenntnis genommen wird. Trotz vieler gegenteiliger Behauptungen sind historische Objekte in Ausstellungen in einer Reihe von Fällen lediglich schmückendes, jedoch kaum beachtetes Beiwerk. Auch für diese Beobachtung gibt die Planck-Ausstellung der MPG in Berlin ein unrühmliches Beispiel ab. Angesichts der Fülle der Texte und des Bildmaterials hätte man sich die wenigen historischen Exponate ehrlicherweise auch ganz sparen können. Sie standen verloren in der Ausstellung, trugen kaum zur dargestellten Geschichte bei, geschweige denn wurden sie als kulturelle Artefakte in ihrer historischen Bedeutung ernst genommen. Den Blick vorrangig auf die Werbung für die eigene Institution gerichtet, blieb offensichtlich für die intensive Objektsuche in den Depots der Museen und Archive keine Zeit beziehungsweise es wurden dieser Aufgabe zu wenig Aufmerksamkeit und Ressourcen gewidmet.

Der hier bemängelte Austausch zwischen Forschung, Ausstellung und Sammlungen hat selbstverständlich auch Gründe, die innerhalb der Museen liegen. Oft genug ist der Zugang zu Objekten in Depots sehr schwierig. Ich kann nur dazu aufrufen, anzuklopfen, wenn sich die Türen nicht automatisch öffnen, gerne auch hörbar. Auf der anderen Seite sitzen vielleicht Kuratorinnen oder Kuratoren, die bereitwillig ihre Türen aufsperren, obwohl sie sich auch innerhalb ihrer Institutionen angesichts der vielen Aufgaben, die ebenfalls von ihnen eingefordert werden, rechtfertigen müssen. Allerdings kann nicht selbstverständlich davon ausgegangen werden, dass sie eine wissenschafts- oder technikhistorische Ausbildung vorweisen können. Auch dies ist eine Folge der skizzierten Entwicklungen. Es ist zu hoffen, dass in den verschiedenen neu eingerichteten Bachelor- und Masterprogrammen, in denen wissenschafts- und technikgeschichtliche Kenntnisse erworben werden, Museen als potentielle Arbeitgeber ernst genommen werden. Zugleich muss aktiv darauf hingewirkt werden, dass Museen die so ausgebildeten Absolventen auch bei der Stellenvergabe berücksichtigen.

Die hier skizzierte pessimistische Sicht auf die gegenwärtige Entwicklung scheint im Übrigen auch für Museen jenseits der Wissenschaft und Technik zuzutreffen. So formulierte beispielsweise Edelbert Köb, Direktor des $\mathrm{Mu}$ seums Moderner Kunst Stiftung Ludwig Wien, auf dem Österreichischen Museumstag 2007 knapp zum Sammlungs- und Bildungsauftrag des Museums in Zeiten der Eventkultur: „Trotz allgemeiner Aufmerksamkeit befindet 
sich das Museum als Ort der Sammlung heute leider in einer Baisse, das Sonderausstellungswesen aber in einer Hausse. [...] Der kulturelle Auftrag des Museums als Ort des Sammelns, Bewahrens und Forschens tritt doch zunehmend in den Hintergrund des allgemeinen Interesses." Auch argumentiert er "gegen die schleichende Ökonomisierung und Popularisierung der wissenschaftlichen Bildungseinrichtung Museum" und ist sich dabei der begrenzten Reichweite der Argumente in der heutigen Situation bewusst. Auf die Museen der Wissenschaft und Technik gewendet bedeutet dies: Nur wenn die Vertreterinnen und Vertreter der Fachwissenschaften gemeinsam mit denen der Wissenschafts- und Technikgeschichte Museen stärker unterstützen und sich bemühen, diese in ihrer Existenz zu sichern, können zukünftig noch materielle Quellen der Wissenschaft und Technik bewahrt werden. Großzügige Appelle und Aufrufe, wissenschafts- oder technikhistorisch bedeutende Artefakte an Museen zur Bewahrung zu geben, werden sonst ins Leere laufen. Ohne solche Anstrengungen, aktiv Einfluss auf den Kurs der Museen zu nehmen, wird es möglicherweise bald keine Museen mehr geben.

Christian Sichau

Deutsches Museum

80306 München

E-Mail: c.sichau@deutsches-museum.de 